\title{
ARTE Y DISIDENCIA EN LA SOCIEDAD FRAGMENTADA
}

\author{
Ion Andoni del Amo Castro ${ }^{1}$ \\ Universidad del País Vasco / Euskal Herriko Unibertsitatea. Dpto. Teoría e Historia de la Educación. \\ IkasGuraikerketataldea
}

\begin{abstract}
Arkaitz Letamendia Onzain²
Universidad del País Vasco / Euskal Herriko Unibertsitatea. Dpto. Ciencia Política. Parte Hartuz ikerketa taldea
\end{abstract}

\section{Jason Diaux González}

Universidad del País Vasco / Euskal Herriko Unibertsitatea. Dpto. Sociología II

\section{Resumen}

La cuestión de si desde el terreno del arte sigue siendo todavía posible la disidencia remite a su significación política, a su capacidad de transformar el entorno. Para indagar en el papel del arte y la disidencia, en este artículo profundizamos en qué tipo de arte y prácticas culturales conectan con disidencias con incidencia política. Proponemos para ello contextualizar históricamente los dos grandes marcos culturalesy artísticos del último siglo, el moderno y el postmoderno. A través de estos marcos abordamos cronológicamente el papel de las vanguardias artísticas modernas, las subculturas musicales y los youtubers digitales en las actuales sociedades, y sus potencialidades de transformación política. Concluimos que la disidencia en el arte, en unas sociedades fragmentadas social y culturalmente como las de hoy, remite especialmente a su reconstrucción colectiva.

Palabras Clave: ARTE; DISIDENCIA; MÚSICA; POSTMODERNIDAD; FRAGMENTACIÓN SOCIAL

\section{ART AND DISSIDENCE IN THE FRAGMENTED SOCIETY}

\section{Abstract}

The question of the possibility of dissidence in the field of art refers to its political significance, its ability to transform the social environment. To investigate the role of art and dissidence, in this article we delve into what kind of art and cultural practices connect with dissidences with political incidence. We propose to contextualize historically the two great cultural and artistic frameworks of the last century, the modern and the postmodern. Through these frameworks, we chronologically address the role of modern artistic avant-gardes, musical subcultures and digital youtubers in current societies, and their potential for political transformation. We conclude that dissidence in art, in socially and culturally fragmented contemporary societies, refers especially to its collective reconstruction.

Keywords: ART; DISSIDENCE; MUSIC; POSTMODERNITY; SOCIAL FRAGMENTATION

Del Amo Castro, Ion Andoni, Arkaitz Letamendia Onzain \& Jason Diaux

González. 2018. "Arte y disidencia en la sociedad fragmentada".

AusArt6(2): 23-34. D0I:10.1387/ausart.20342

\section{AUSART}




\section{INTRODUCCIÓN}

La cuestión de si desde el terreno del arte sigue siendo todavía posible la disidencia remite, en cierto modo, a su significación política. La capacidad de generar aperturas simbólicas y de transformar el entorno, de incidir en el espacio vital sociocultural, es el acto de significación política por excelencia.

Para indagar en el papel del arte y la disidencia, profundizamos en la cuestión de qué tipo de arte y prácticas culturales son las que conectan con disidencias con incidencia política, en relación a un contexto sociocultural concreto. Se trata, por tanto, de una reflexión teórica elaborada a partir de una lectura sintomática en el marco de un análisis coyuntural respecto de las condiciones sociales, económicas, culturales y políticas (Hall \& Jefferson 2006; Griffin 2010).

Así, proponemos una breve contextualización histórica de los dos grandes marcos culturalesdel último siglo, el moderno y el postmoderno. Nos centraremos en éste último, sus condiciones estructurales y, en consecuencia, las posibilidades y límites actuales de disidencia política, ilustradas a través de dos ejemplos de caso.

\section{ACABAR CON EL ARTE (BURGUÉS)}

Si la modernidad posee un significado, es éste: lleva consigo, desde el principio, una negación radical, el dadá, ese acontecimiento que tuvo lugar en un café de Zúrich. (Lefebvre citado en Marcus 2005, 32)

El proyecto de la modernidad unido al capitalismo genera malestar desde sus inicios. Boltanski y Chiapello (2002) agrupan esas fuentes de indignación en dos categorías que denominan crítica artística y crítica social. La primera pone en primer plano la pérdida de sentido, especialmente de lo bello y grandioso, derivada de la estandarización y mercantilización de los objetos, las obras de arte, e incluso los seres humanos. A ello opone la libertad del artista, su rechazo a una contaminación de la estética por la ética, su desprecio por toda forma de sometimiento en el tiempo y en el espacio, incluso por todo tipo de 
trabajo. Una expresión ejemplar sería Baudelaire, y el modelo de vida bohemio.

La crítica social, por su parte, se inspira en los socialistas y marxistas, y hace referencia a fuentes de indignación como el egoísmo y la miseria crecientes: "Apoyándose en la moral y, a menudo, en una temática de inspiración cristiana, la crítica social rechaza, a veces con violencia, el inmoralismo o el neutralismo moral, el individualismo, inclusive el egoísmo o egotismo, de los artistas" (Boltanski \& Chiapello 2002, 86).

Ambas críticas pueden, según coyunturas históricas, entrar en tensión o verse asociadas, aumentando su potencialidad. Así, Jameson (1998) recuerda que el modernismo en las artes se relaciona íntimamente con los vientos de cambio que soplan desde los movimientos sociales radicales. Casanovas (2012, 19) apunta que "el arte y la política no son dos realidades permanentemente separadas que se deban poner en relación, sino que (cuando hay propiamente 'arte' o propiamente 'política') estamos hablando de un orden de experiencia análogo", una reconfiguración de la división de lo sensible. En la misma línea, Eyerman y Jamison (1998) señalan que mediante la combinación de cultura y política los movimientos sociales reconstituyen a ambos, proporcionan un contexto interno político e histórico para la expresión cultural, y ofrecen, a su vez, los recursos de la cultura a los repertorios de acción política. Podemos verlo a lo largo del siglo XX.

\subsection{Cambiar la vida, transformar el mundo}

La I Guerra Mundial supondrá el momento "cuando, definitivamente, la burguesía perderá su 'inocencia' como clase supuestamente representante del progreso y la libertad" (Casanovas 2012, 39). Es el contexto de las primeras vanguardias político-culturales del siglo XX:

La crítica que inauguran las vanguardias no es una 'crítica inmanente' (a corrientes y escuelas artísticas precedentes), sino una crítica del papel que juega el arte como institución dentro de la sociedad burguesa. Crítica de los modos en que el arte es producido y distribuido dentro de dicha sociedad y su relación con la vida cotidiana.

(Casanovas, 2012: 31) 
Así, para dadá, la cultura occidental es una solemne farsa que ha derivado en la carnicería que asola Europa; debe ser destruida para empezar de cero (Granés 2011). Constructivistas rusos y suprematistas, por su parte, sostendrán el proyecto -moderno- de crear un 'ser humano nuevo' a través del Arte, e intentarán realizarlo en el horizonte abierto por la revolución rusa. El futurismo italiano encontrará en los nuevos desarrollos tecnológicos, en las máquinas, y en la capacidad 'regeneradora' de la guerra, la utopía de una sociedad nueva.

Y el Arte burgués será derribado. Pero eso no calmará a los insatisfechos, porque la victoria parece ser la de una cultura de masas mercantilizada, aunque traiga dentro de sí trazos de lo popular, en un doble proceso de reconocimiento y expropiación (Martín-Barbero, 1993). La cultura de masas mercantilizada marca un rasgo de semejanza -en el cine, en la radio, en la televisión, en las revistas-, y se afianza a través de una industria cultural que concentra los centros de producción, dispersa la recepción y estandariza los productos de consumo (Horkheimer \& Adorno1947). La crítica se dirige entonces contra el exceso de institucionalización, mercantilización y uniformización, que impide una expresión espontánea (Marcuse 1964). Especialmente para los situacionistas (Debord 1967), el arte, en esas condiciones, estaba definitivamente muerto y solo cabía enterrar su cadáver, se había transformado en mero espectáculo, en reinos de fantasía que sustituían a la vida real. El arte debía ser la vida cotidiana.

\section{A FAVOR DE ESTAR EN CONTRA EN LA POSTMODERNIDAD}

En el marco del capitalismo tardío, sin embargo, emerge una nueva lógica cultural, la postmodernidad, en la que la diferencia se impone progresivamente, frente a la uniformidad de la cultura de masas, como garantía de legitimidad (Yúdice 2002).

Es, en gran medida, el resultado de las batallas y reivindicaciones de los años 60 y 70, en las que la cultura se había entendido como un 'terreno de lucha', según la teoría gramsciana, y supone grandes dosis de democratización de la cultura. Pero mientras que para la modernidad crítica la sensación de que todo se ha hecho ya, que la invención formal o estilística es imposible, derivaba en la idea de que el arte ha terminado y la crítica lo sustituye, para la postmoder- 
nidad, por contra, las cosas no ofrecen este aspecto, y significa, simplemente, que "todo vale" (Jameson 1998, 247).

Setenta años de revoluciones culturales dieron sus frutos. El germen anarquista y libertario que llegó con el futurismo y el dadaísmo (...) sobrevivió a la muerte de estos movimientos y se fue transmitiendo (...) de los dadaístas a los músicos, de los músicos a los poetas beat y de los poetas beat a los yippies, hasta salir de los socavones y convertirse en el lenguaje de los medios de comunicación y en la nueva sensibilidad de los jóvenes de las últimas cuatro décadas.

Pero el triunfo significó también su derrota. Una vez que el discurso anárquico de la vanguardia fue asimilado por la sociedad y enaltecido por el mercado, su gesto transgresor se convirtió en una pirueta estereotipada. Sin la vitalidad que proporcionaba luchar contra una sociedad conservadora y tozuda, y sin un horizonte de transformación social, la revuelta se convirtió en una maniobra circense para mantener el mercado del arte al rojo vivo y los precios por las nubes. Ya no había nada que derrotar.

(Granés 2011, 418)

Ésta es una inversión fundamental respecto de los modernismos artísticos de las vanguardias, que se distinguen por su hostilidad al mercado (Jameson 1998).

Las lógicas postmodernas y multiculturales contemporáneas corren en paralelo a la fragmentación sociocultural. Y en ese marco se resiente la capacidad de producir aperturas simbólicas, es decir,de visualizar una imagen, una explicación del mundo, y otros mundos alternativos (Jameson 1998). Así, la provocación o la transgresión, que habían sido una táctica habitual, quedan problematizadas, "ya que no solamente se reciben con la mayor complacencia, sino que ellos mismos se han institucionalizado e incorporado a la cultura oficial de la sociedad occidental" (Jameson 1995, 17).

Y ello indica al mismo tiempo que algunas de nuestras más apreciadas y venerables ideas radicales sobre la naturaleza de la política cultural pueden, en consecuencia, resultar anacrónicas. No importa lo distintas que hayan sido estas concepciones-desde los tópicos de la negatividad, la oposición y la subversión, hasta los de la crítica y la reflexión-, todas ellas compartían un mismo presu- 
puesto -fundamentalmente espacial- que resume a la perfección la expresión, no menos venerable, "distancia crítica'. En relación con la política cultural, no existe hoy una sola teoría de izquierdas que haya sido capaz de prescindir de la idea, expresada de un modo u otro, de una distanciación estética mínima, es decir, de la posibilidad de situar la acción cultural fuera del ser compacto del capital y utilizarla como un arquimedeano punto de apoyo desde el que atacar al propio capitalismo.

(Ibíd., 107)

Y es en este complicado contexto sociocultural, sumado a los cambios tecnológicos, en el que vamos a tratar de analizar las posibilidades y límites de la disidencia en el arte. Lo haremos a través de dos 'trazos' ejemplo.

\section{UN PAR DE TRAZOS ¿DISIDENTES?}

Que todo el mundo se convierta en artista. Era la propuesta de Lautréamont, convertida en ariete de acción político-cultural por parte de las vanguardias artísticas, queriendo insertar el arte en la vida cotidiana. Era también el sueño de los hippies, la consigna DIY (Do It Yourself, o 'Hazlo tú mismo') y la praxis autogestionaria frente a la industria cultural de masas.

La era digital abre hoy nuevas posibilidades. El desarrollo tecnológico permite que los sujetos puedan construir sus propias redes de comunicación, autocomunicar (Castells 2009). Así, a través de plataformas como Youtube los contenidos generados por los usuarios, basados en características como expresividad, performatividad y colaboración, promueven la participación política (Ostman 2012); y hacen emerger artefactos culturales audiovisuales que transitan en redes digitales.

El sueño de las vanguardias se acerca, pero se aleja al tiempo en una situación estructural de creciente mercantilización (Fuchs \& Sandoval 2014; Kim, 2012), y en la problemática lógica cultural postmoderna. Para indagar estas posibilidades y límites, abordamos a continuación dos casos. 


\subsection{TODAVÍA, UNA CANCIÓN}

La disidencia también se canta. En el siglo XX la conjunción de movimientos de protesta y movilización cultural constituye un fértil terreno para la (re)creación cultural y para la transmisión de mensajes y visiones del mundo alternativas. La música resulta un recurso cultural muy destacado, por su especial capacidad de movilizar sentimientos y emociones (Eyerman \& Jamison 1998; Larrinaga 2016).

Tal es el caso de las subculturas juveniles -punk, reggae, rap- desde los años 70 (Hall \& Jefferson 2006; Hebdige 1979). Surgen de ámbitos autónomos de comunicación y socialización, donde se produce un 'discurso oculto' que expresa disidencia y resistencia frente a las formas existentes de dominación (Scott 2003), y permiten la construcción de espacios cotidianos que ayudaban al crecimiento de la autonomía cultural y autoestima. En estos lugares se incentivan filosofías como el DIY que, en la línea de las vanguardias, inciden en la capacidad de creación espontánea y directa de los sujetos y artistas, al margen de la gran industria cultural de masas. Lo vemos en EuskalHerria en los ámbitos autónomos y el conjunto de gaztetxes, txosnas, fanzines, bandas musicales y radios libres que emergen en los años 80 (Del Amo 2016). Los ruidos adquieren significación política, inseparables del conflictivo ambiente sociopolítico del momento.

Sin embargo, en el marco de la postmodernidad más reciente la capacidad de la música para movilizar la protesta también se ve comprometida, registrándose cambios en su significado social. Actualmente predominan los elementos indiferenciadores y los 'grandes eventos' (festivales, etc.), lo que apunta al agotamiento, en unas sociedades crecientemente fragmentadas y desarticuladas, de las construcciones identitarias diferenciadoras, de las 'tribus' subculturales (Del Amo Letamendia \& Diaux 2016).

Asimismo, en el nuevo ciclo de protesta internacional abierto en 2011 la movilización de la música no parece tener tanta importancia como en ciclos anteriores (Del Amo 2016). Las voces de protesta se expresan más por otras vías -redes sociales, por ejemplo- y no tienen una banda sonora determinada. Sin embargo, la represión sí la tiene, al menos en el Estado español de la década de los 2010: el rap. Asistimos a procesos penales que culminan en condenas a raperos por el contenido de sus canciones, como Pablo Hasel, Valtonyc, o La Insurgencia; y que suceden en un escenario de represión creciente, como reflejan las condenas a twitteros (entre ellos elvocalista del grupo rap-metal Def Con Dos) o el encarcelamiento de políticos soberanistas catalanes. 
Las condenas a los raperos, sin embargo, han tenido un efecto movilizador. Han propiciado la movilización solidaria de la música como elemento de reivindicación. Muestra de ello es el tema coral de comienzos de 2018 realizado por un conjunto de raperas y raperos, que se solidariza con los condenados, denuncia la represión y clama por la libertad de expresión. Se trata del videoclip Los borbones son unos ladrones, apoyado por la productora independiente catalana Propaganda pelfet!, y que en siete meses supera los 3,7 millones de visualizaciones ${ }^{3}$. Un aspecto fundamental, que lo convierte en una expresión de disidencia política que trasciende lo estético, es el de la implicación colectiva (simbólica y factual) de sus autores con la reivindicación planteada. Las y los artistas cantan la libertad de expresión y denuncian la represión, aún si son ellas con sus letras sus próximas víctimas. EI DIY surgido en los años 70 -ámbitos autónomos de socialización política, subculturas musicales- aún pervive, canta en tonos de disidencia, y se hace oír.

\subsection{EL DIY EN LA ERA DIGITAL: LOS YOUTUBERS}

La era digital tiene hoy su propio y característico DIY: los youtubers. En un contexto de aumento de la precariedad en diversos ámbitos de lo social (Heidkamp \& Kergel 2017), y modos fragmentados e individualizados de interacción social, la praxis autogestionaria adquiere nuevos formatos. Imbuida de una lógica más individualista, la anterior espontaneidad y creación del DIY, surgida en ámbitos autónomos de socialización, se expresa hoy mediante unos youtubers que operan y crean artefactos audiovisuales directamente. Sin intermediarios, desde sus casas, pero conectados a redes de comunicación globales.

Según plantea Castells (2009) las redes sociales digitales pueden actuar como herramientas de autocomunicación de la disidencia; incluso ésta puede aún luchar por la reapropiación tecnológica para disputar la hegemonía comunicativa (Del Amo, Letamendia \& Diaux 2014). Pero desde el momento en que la infraestructura material no ha de ser construida colectivamente-como en el DIY autónomo de las radios libres, la música y los fanzines- la implicación comunitaria tiende a desaparecer, y con ello la significación política.

Recientes publicaciones indagan en esta cuestión: Lovelock (2017) apunta a los ideales neoliberales que actualmente encarnan las subjetividades youtubers, incluso cuando representan sexualidades no-normativas; mientras que Aran-Ramspott, Fedele y Tarragó (2018) subrayan la capacidad de los youtubers de constituirse en referentes de una cultura digital juvenil desarrollada 
en un ambiente mediatizado, y que forma parte de las oportunidades y servidumbres del sistema neoliberal contemporáneo. El resultado es un producto digital, consumido en redes sociales, que 'estetiza' la resistencia y la despoja de capacidad de transformación profunda (Letamendia 2017). La disidencia aquí se consume, y marca un estilo -rebelde, rompedor- que se suma a otros en ese supermercado neoliberal, global y digital.

En la era digital los lugares de disidencia con incidencia política se expresan en 'grandes eventos' más materiales que culturales -plazas ocupadas en el Estado español, colegios electorales defendidos en Catalunya, carreteras cortadas en Francia-, que tienden a (re)construir, a veces de modo espasmódico, las relaciones sociales directas y comunitarias que la postmodernidad y las propias TIC han diluido. Se constituye así la paradoja de la búsqueda de la conexión (directa, física, emocional) en un mundo ultraconectado digitalmente. Sin esta conexión humana, sin una comunidad directa que la genere, la disidencia política y su arte se tornan improbables.

\section{ACABAR (CON ESTA REFLEXIÓN)}

\subsection{Que el arte sea comunal}

Los desarrollos tecnológicos, que facilitan la autocomunicación, acercan la utopía de las vanguardias de que (casi) todo el mundo se convierta en artista: impulsan la creatividad y los intercambios, el ethos DIY. Pero el hacérselo uno mismo, en tanto que másliteral y en el marco cultural postmoderno del "todo vale", paralelamente aleja la utopía, la vacía de contenido político. Impulsa el individualismo antes que la autonomía cultural y, recuperando la sentencia situacionista, queda subsumido en el espectáculo (digital); en terminología coloquial y postmoderna, deviene en postureo.

Romper la dicotomía entre cultura activa y pasiva, colectivizando y desmercantilizando los procesos productivos, enraizando el arte y la creatividad en la vida cotidiana, era el proyecto de cambio (y destrucción creativa) de las vanguardias modernas. En unas sociedades como las actuales, fragmentadas social y culturalmente, el sentido social del arte podría apuntar en la dirección de fortalecer los elementos colectivos, comunitarios y de solidaridad de la crítica social. 


\section{2. ¿Y LAS ESTRUCTURAS DE PODER?}

En el contexto general del complicado papel del arte político contemporáneo, las estructuras de poder siguen siendo un elemento relevante: cuando hay algo directamente a combatir, una amenaza material, la dimensión política de la creación cultural reaparece. Ya no se trata de una simple pirueta estética. Lo hemos visto en el caso del Estado español, donde la represión ejercida recientemente sobre algunos artistas de escenas rap revitaliza el sentido político, movilizador, disidente, de la música.

\subsection{El arTE dE LA SOCIEDAd FRAGMENTADA}

El terreno de las prácticas artísticas, en efecto, ha servido de lugar para el desarrollo de cierta subjetividad crítica, espacios de autonomía, resistencia y lucha ideológica. La postmodernidad, sin embargo, ha complicado su papel transgresor, y la penetración de la lógica capitalista ha socavado su propia autonomía. A la pregunta de si desde el terreno del arte sigue siendo todavía posible la disidencia, hemos pretendido aportar que ésta debe situarse en relación a las propias estructuras sociales y culturales. Trocear y destruir el arte como acto de disidencia ha de suponer, en unas sociedades fragmentadas social y culturalmente como las actuales, unirse a la labor titánica de su reconstrucción colectiva. La luz de guía que pueda iluminar hoy será una llamacolectiva, que temple el malestar de la fragmentación. Y, como siempre, que responda a la oscuridad provocada desde el poder.

\section{Referencias}

Aran-Ramspott, Sue, Maddalena Fedele \& Anna Tarragó. 2018. "Funciones sociales de los youtubers y su influencia en la preadolescencia". Comunicar 57: 71-80. DOI: https://doi. org/10.3916/C57-2018-07

Boltanski, Luc \& Ėve Chiapello. 2002. El nuevo espíritu del capitalismo. Traducción Marisa Pérez Colina, Alberto Riesco Sanz \& Raúl Sánchez Cedillo; edición de Carlos Prieto del Campo. Madrid: Akal

Casanovas, Marc. 2012. Organizar el rechazo: Vanguardias culturales y política revolucionaria. Madrid: Izquierda Anticapitalista

Castells Oliván, Manuel. 2009. Comunicación y poder. Madrid: Alianza

Debord, Guy. (1967) 2003. La sociedad del espectáculo. Prólogo, traducción y notas de José Luis Pardo. Valencia: Pre-Textos 
Del Amo Castro, Ion Andoni. 2016. Party \& Borroka: Jóvenes, músicas y conflictos en Euskal Herria. Tafalla: Txalaparta

Del Amo Castro, Ion Andoni, Arkaitz Letamendia Onzain \& Jason Diaux. 2014. "Nuevas resistencias comunicativas: La rebelión de los ACARP”. Revista Latina de Comunicación Social 69: 307-29. DOI: https://doi.org/10.4185/RLCS-2014-1013

— . 2016. “¿El declive del significado social de la música?”. Revista Crítica de CiênciasSociais109: 11-32. DOI: https://journals.openedition.org/rccs/6189

Eyerman, Ron \& Andrew Jamison. 1998. Music and social movements. Cambridge MA: Cambridge University

Fuchs, Christian \& Marisol Sandoval, eds. 2014. Critique, social media and the information society. New York: Routledge

Granés Maya, Carlos. 2011. El puño invisible: Arte, revolución y un siglo de cambios culturales. Madrid: Santillana

Griffin, Christine Elizabeth. 2010. "The trouble with class: Researching youth, class and culture beyond the 'Birmingham School'”. Journal of Youth Studies, 14(3): 245-59. DOI: https://doi. org/10.1080/13676261.2010.533757

Hall, Stuart \& Tony Jefferson, eds. 2006. Resistance through rituals: Youth subcultures in postwar Britain. New York: Routledge

Hebdige, Dick. 1979. Subculture: The meaning of style. London: Routledge

Heidkamp, Birte \& David Kergel, eds. 2017. Precarity within the Digital Age: Media change and social insecurity. Wiesbaden: Springer. DOI: https://doi.org/10.1007/978-3-658-17678-5

Horkheimer, Max \& Theodor W. Adorno. (1947) 1994. Dialéctica de la Ilustración: Fragmentos filosóficos. Introducción y traducción de Juan José Sánchez. Madrid: Trotta

Jameson, Fredric. 1995. El postmodernismo o La lógica cultural del capitalismo tardío. Traductor José Luis Pardo Torio. Barcelona: Paidós

- . 1998. Teoría de la postmodernidad. Traducción de Celia MontolíoNicholson\& Ramón del Castillo. Madrid: Trotta

Kim, Jin. 2012. "The institutionalization of YouTube: From user-generated content to professionally generated content”. Media Culture \& Society, 34(1): 53-67. DOI https://doi. org/10.1177/0163443711427199

Larrinaga Arza, Josu. 2016. Euskal musika kosmikoak: Euskal musika popularra gizartearen isla eta aldatzailea. Mungia: Baga-Biga

Letamendia Onzain, Arkaitz. 2017. "Towards the aestheticisation of the resistances in the Digital Age? A critical approach". En Precarity within the Digital Age: Media change and social insecurity, edited by Birte Heidkamp \& David Kergel, 135-49. Wiesbaden: Springer. DOI: https://doi.org/10.1007/978-3-658-17678-5_9

Lovelock, Michael. 2017. "'Is every YouTuber going to make a coming out video eventually?' YouTube celebrity video bloggers and lesbian and gay identity". Celebrity Studies 8(1): 87-103. DOI: https://doi.org/10.1080/19392397.2016.1214608

Marcus, Greil. 2005. Rastros de Carmín: Una historia secreta del siglo XX. Traducción de Damián Alou. Barcelona: Anagrama

Marcuse, Herbert. (1964) 2016. El hombre unidimensional. Traducción Antonio Elorza. BarceIona: Planeta 
Martín-Barbero, Jesús. 1993. De los medios a las mediaciones: Comunicación, cultura y hegemonía. México: Gustavo Gili

Ostman, Johan. 2012. "Information, expression, participation: How involvement in user-generated content relates to democratic engagement among young people". New Media \& Society, 14(6): 1004-21. DOI: https://doi.org/10.1177/1461444812438212

Scott, James C. 2003. Los dominados y el arte de la resistencia. Traducción de Jorge Aguilar Mora. Tafalla: Txalaparta

Yúdice, George. 2002. El recurso de la cultura: Usos de la cultura en la era global. Traducción, Gabriel Ventureira \& Desiderio Navarro. Barcelona: Gedisa

\section{Notas}

${ }^{1}$ Investigación desarrollada en el marco del programa de especialización de doctores de la UPV/EHU.

${ }^{2}$ Investigación desarrollada en el marco del programa postdoctoral del Gobierno Vasco.

${ }^{3}$ https://www.youtube.com/watch?v=ySqxLQ-UsNo. Acceso 25 oct. 2018 